\title{
CONVEXITY AND TIGHTNESS FOR RESTRICTIONS OF HAMILTONIAN FUNCTIONS TO FIXED POINT SETS OF AN ANTISYMPLECTIC INVOLUTION
}

BY

\author{
J. J. DUISTERMAAT ${ }^{1}$
}

\begin{abstract}
ABSTRACr. The Kostant convexity theorem for real flag manifolds is generalized to a Hamiltonian framework. More precisely, it is proved that if $f$ is the momentum mapping for a Hamiltonian torus action on a symplectic manifold $M$ and $Q$ is the fixed point set of an antisymplectic involution of $M$ leaving $f$ invariant, then $f(Q)=f(M)=$ a convex polytope. Also it is proved that the coordinate functions of $f$ are tight, using "half-turn" involutions of $Q$.
\end{abstract}

1. Introduction. Recently the theorem of Kostant [10], on convexity of certain projections of complex flag manifolds, has been generalized to a Hamiltonian framework by Guillemin and Sternberg [7], and, independently, by Atiyah [2]. However, Kostant proved his theorem for the real flag manifolds as well, and it is the first purpose of this paper to show that also this real version has a generalization in a Hamiltonian setting. More specifically, let $M$ be a compact connected smooth manifold of dimension $2 n$, provided with a symplectic form $\sigma$. Let $T$ be a torus acting on $M$ in a Hamiltonian way, with Hamiltonian functions $f_{X}, X \in \mathfrak{t}$, and momentum mapping $f: M \rightarrow \mathrm{t}^{*}$. Here $\mathrm{t}$ is the Lie algebra of $T$ and $\mathrm{t}^{*}$ its dual, see $\S 2$ for more details. Furthermore, let $\tau$ be a smooth involution of $M$ such that $\tau^{*} \sigma=-\sigma$ and such that $f_{X} \circ \tau=f_{X}$ for all $X \in \mathrm{t}$. (One can always arrange the latter condition by passing to a suitable subtorus $T_{0}$ of $T$, the new momentum mapping then being equal to $f$ followed by the natural projection $\mathrm{t}^{*} \rightarrow \mathrm{t}_{0}^{*}$.) Let $Q$ be the fixed point set of $\tau$, which we assume to be nonvoid. Then $f(Q)=f(M)$, the convex hull of finitely many points in $\mathrm{t}^{*}$. For a more detailed description of the extremal points of $f(Q)=f(M)$, see Theorem 2.5 and formula (2.32). The proof follows the pattern of Guillemin and Sternberg [7], which in turn was inspired by Heckman [8].

If $M$ is a Kähler manifold, then a more refined result is true in terms of gradient flows, this will be discussed in $\S 4$.

Secondly, Atiyah [2], following Frankel [6], observed that the Hamiltonian functions $f_{X}, X \in \mathrm{t}$, are tight in the sense that the sum of the Betti numbers of $M$ is equal to the sum of the Betti numbers of the critical set of $f_{X}$. In the case of isolated critical points this implies that $f_{X}$ is a Morse function on $M$ with the minimal number of critical points. This too generalizes a known result for complex flag manifolds, but

Received by the editors December 7, 1981 and, in revised form, January 21, 1982.

1980 Mathematics Subject Classification. Primary 53C15; Secondary 53C55, 58F05, 55M20.

' Partially supported by NSF Grant MCS 80-23356, while visiting the University of California at Berkeley. 
which actually is true for the real flag manifolds as well, due to Takeuchi and Kobayashi [13], see also Divistermaat, Kolk and Varadarajan [3, §4]. In §3 it will be shown that also the tightness generalizes to our setting. The theorem is that $\operatorname{dim} H^{*}(Q ; \mathbf{Z} / 2 \mathbf{Z})=\operatorname{dim} H^{*}\left(C_{X} ; \mathbf{Z} / 2 \mathbf{Z}\right)$ if $C_{X}$ is the critical set of the function $f_{X} \mid Q, X \in \mathrm{t}$.

Finally in $\$ 5$ we describe how the real flag manifolds fit into the framework described above.

It is a pleasure for me to thank Michael Atiyah for his suggestion, made to me at the Arbeitstagung in Bonn in June 1981 (and in [2]), that the real flag manifolds really should be treated as the fixed point set of an involution in a symplectic manifold. I also thank John Millson, resp. Alan Weinstein for some discussions at UCLA, resp. Berkeley, which stimulated me further.

2. Convexity. We recall that $(M, \sigma)$ is a compact connected symplectic manifold with a Hamiltonian action of a torus $T$ on it. That is, there is a linear map $X \mapsto f_{X}$ from the Lie algebra $t$ of $T$ to the space of smooth functions on $M$, such that

(2.1) For each $X \in \mathfrak{t}$, the infintesimal action $\tilde{X}$ of $X$ on $M$ is equal to the Hamilton vector field of the function $f_{X}$, and

(2.2) The functions $f_{X}, X \in \mathrm{t}$, are in involution.

In formula, (2.1) reads

$$
\tilde{X}\lrcorner \sigma=-d f_{X}, \quad X \in \mathfrak{t},
$$

whereas assuming this, (2.2) is equivalent to

$$
\tilde{X}\lrcorner d f_{Y}=0 \text { for all } X, Y \in \mathrm{t},
$$

that is, $f_{Y}$ is constant along the $T$-orbits in $M$.

The mapping $f: M \rightarrow \mathrm{t}^{*}$, defined by

$$
\langle X, f(m)\rangle=f_{X}(m), \quad m \in M, X \in \mathrm{t},
$$

is called the momentum mapping of the Hamiltonian $T$-action.

The next ingredient which we introduce is a smooth map $\tau: M \rightarrow M$ which is an involution of $M$, that is

$$
\tau \circ \tau=\text { identity on } M,
$$

and which is antisymplectic, that is

$$
\tau^{*} \sigma=-\sigma .
$$

We will assume that the Hamiltonian $T$-action and the involution $\tau$ are related to each other by the condition that the functions $f_{X}$ are $\tau$-invariant, that is

$$
\tau^{*} f_{X}=f_{X} \text { for all } X \in \mathrm{t} .
$$

Assuming (2.5), this is equivalent to

$$
\tau^{*} \tilde{X}=-\tilde{X}, \quad X \in \mathrm{t},
$$

in view of $\left(2.1^{\prime}\right)$. In turn, $(2.7)$ is equivalent to

$$
\tau \tilde{g} \tau^{-1}=\tilde{g}^{-1}, \quad g \in T,
$$

if $\tilde{g}$ denotes the action of $g$ on $M$. That is, $\tau$ maps $T$-orbits to $T$-orbits, but at the same time reverses the time on the orbits of the 1-parameter subgroups of $T$. 
If $G$ is a compact group acting by smooth mappings on a manifold $M$, then averaging over $G$ of an arbitrary Riemannian metric on $M$ leads to a $G$-invariant Riemannian metric $\beta$ on $M$. If $m$ is a fixed point for the $G$-action, then the exponential map, centered at $m$, with respect to $\beta$, intertwines the linear action of $G$ on $T_{m} M$ with the local action of $G$ around $m$. That is, the $G$-action is linear orthogonal on suitable local coordinates. In particular the fixed point set for the action of any subset of $G$ has finitely many components, each of which is a closed smooth submanifold of $M$.

We assume that the fixed point set of $\tau$ in $M$,

$$
Q=\{m \in M ; \tau(m)=m\},
$$

is nonvoid. It has finitely many smooth, compact, connected components. From (2.5) we obtain that if $m \in Q$, then both $T_{m} M=\operatorname{Ker}(D \tau(m)-I)$ and

$$
P_{m}=\operatorname{Ker}(D \tau(m)+I)
$$

are isotropic subspaces for $\sigma_{m}$. Because also

$$
T_{m} M=T_{m} Q \oplus P_{m}
$$

the conclusion is that $T_{m} Q$ and $P_{m}$ are Lagrange subspaces of $T_{m} M$. That is, $Q$ is a Lagrange submanifold of $M$, and the $P_{m}, m \in Q$, form a Lagrange subbundle of $T_{Q} M$, complementary to $T Q$. In fact, Meyer [11] showed that there is an open $\tau$-invariant neighborhood $U$ of $Q$ in $M$ and a symplectic diffeomorphism $\Phi$ from $U$ onto an open neighborhood $V$ of the zero section in $T^{*} Q$, such that $\Phi \tau \Phi^{-1}$ maps $p \in\left(T_{q} Q\right)^{*} \cap V$ to $-p$, for all $q \in Q$.

Another rigidity property of compact Lie group actions on a compact manifold $M$ is that there are only finitely many orbit types (Mostow [12], Yang [15]). In the case of the action of a torus $T$ this means that there are only finitely many possibilities for the stabilizer groups

$$
T_{m}=\{g \in T ; g(m)=m\},
$$

as $m$ ranges over $M$. This fact will be used both in the proof of the convexity and of the tightness theorem mentioned in the introduction.

2.1. Lemma. Let $m \in Q, X \in \mathrm{t}$. Then $d\left(f_{X} \mid Q\right)(m)=0$ implies that $d f_{X}(m)=0$, which in turn is equivalent to the condition that $m$ is a fixed point for the action of $\exp t X, t \in \mathbf{R}$.

Proof. $T_{m} Q=\operatorname{Ker}(D \tau(m)-I)=\left\{u+D \tau(m)(u) ; u \in T_{m} M\right\}$. So using (2.6), we get $0=d f_{X}(m)(u+D \tau(m)(u))=2 d f_{X}(m)(u)$ for all $u \in T_{m} M$.

2.2. Proposition. Let $m \in Q, D(f \mid Q)(m)=0$. Then there exist smooth symplectic local coordinates $q_{j}, p_{j}$ for a neighborhood $U$ of $m$ (zero at $m$ ), such that, for suitable $\omega_{j} \in \mathfrak{t}^{*}$,

$$
f_{X}=f_{X}(m)+\sum_{j=1}^{n} \omega_{j}(X) \cdot\left(q_{j}^{2}+p_{j}^{2}\right) / 2,
$$


and moreover

$$
\tau:(q, p) \mapsto(q,-p)
$$

in $U$. In particular $U \cap Q=\{p=0\}$.

Proof. Although the proof is a combination of the by now standard arguments in Meyer [11] and Guillemin-Sternberg [7, §4], we will give it in some detail for the convenience of the reader. We begin with the result on the tangent level.

In view of Lemma $2.1, m$ is not only a fixed point for $\tau$, but also for the $T$-action. Then the

$$
X_{m}=D \tilde{X}(m): T_{m} M \rightarrow T_{m} M, \quad X \in \mathfrak{t},
$$

form a commuting family of infinitesimally symplectic transformations, antisymmetric with respect to $\beta_{m}$. In particular they are simultaneously diagonalizable over $\mathbf{C}$ with purely imaginary eigenvalues. That is,

$$
T_{m} M \otimes \mathbf{C}=\sum_{\lambda}^{\oplus} E_{\lambda}, \quad X_{m} \mid E_{\lambda}=\lambda(X) \cdot \text { identity on } E_{\lambda} ;
$$

here $X_{m}$ is extended to a complex linear endomorphism of $T_{m} M \otimes \mathbf{C}$ and $\lambda$ is real linear: $\mathrm{t} \rightarrow \mathbf{C}$, taking only purely imaginary values. The complex conjugation maps $E_{\lambda}$ to $E_{\lambda}^{-}=E_{-\lambda}$. Because the $X_{m}$ are infintesimally symplectic, the spaces

$$
E_{\lambda,-\lambda}=\left(E_{\lambda}+E_{-\lambda}\right) \cap T_{m} M
$$

are mutually $\sigma_{m}$-orthogonal. Because they span $T_{m} M$, they form a symplectic vector space decomposition of $T_{m} M$.

Now (2.7) implies that

$$
D \tau(m) \circ X_{m}=-X_{m} \circ D \tau(m),
$$

showing that $D \tau(m)$ maps $E_{\lambda}$ to $E_{-\lambda}$, so it leaves $E_{\lambda,-\lambda}$ invariant. This reduces the problem to the case that all $X_{m}$ are real multiples of one infinitesimally symplectic mapping $J$, the square of which we can take equal to $-I$. Moreover, $J$ maps $T_{m} Q$ to $P_{m}$ and $P_{m}$ to $T_{m} Q$. Now

$$
\langle u, v\rangle=\sigma_{m}(u, J v), \quad u, v \in T_{m} Q,
$$

defines a nondegenerate symmetric bilinear form on $T_{m} Q$. We can write $T_{m} Q=Q_{m}^{+}$ $\oplus Q_{m}^{-},\left\langle Q_{m}^{+}, Q_{m}^{-}\right\rangle=0$, with (2.19) being positive, resp. negative definite, on $Q_{m}^{+}$, resp. $Q_{m}^{-}$. The spaces $Q_{m}^{+}+J\left(Q_{m}^{+}\right)$and $Q_{m}^{-}+J\left(Q_{m}^{-}\right)$are $\sigma_{m}$-orthogonal to each other, leading to a $D \tau(m)$-invariant symplectic vector space decomposition. This reduces the problem to the case that (2.19) is definite.

In the positive case, let $e_{1}, \ldots, e_{n}$ be an orthonormal basis of $T_{m} Q$ with respect to (2.19). Then

$$
(q, p) \mapsto \sum_{j}\left(q_{j} \cdot e_{j}-p_{j} \cdot J e_{j}\right)
$$

is a symplectic mapping, with respect to the symplectic form $\Sigma_{j} d p_{j} \wedge d q_{j}$ on $(q, p)$-space. It intertwines $J$ with the mapping $(q, p) \mapsto(p,-q)$, which, as a linear vector field, is Hamiltonian with Hamilton function

$$
\sum_{j}\left(q_{j}^{2}+p_{j}^{2}\right) / 2
$$


In the negative definite case we get (2.21) with a minus sign in front. This proves the proposition on the tangent level.

For the local normal form we already know that there are local coordinates linearizing $\tau$ and the $T$-action, hence also the vector fields $\tilde{X}, X \in \mathrm{t}$. However, in these coordinates the symplectic form $\sigma$ will in general not be equal to the constant symplectic form $\sigma_{m}=\Sigma_{j} d p_{j} \wedge d q_{j}$. The proof is finished by applying the following version (also known to Weinstein) of the equivariant Darboux lemma of Weinstein [14].

2.3. Lemma. Let $G$ be a compact group acting by diffeomorphisms on a symplectic manifold $(M, \sigma)$, such that $\tilde{g}^{*} \sigma=\varepsilon(g) \cdot \sigma, \varepsilon$ a continuous homomorphism: $G \rightarrow$ $\{-1,+1\}$. Let $m$ be a fixed point for the $G$-action, $\sigma^{\prime}$ another smooth symplectic form defined on a neighborhood of $m$, such that $\tilde{g}^{*} \sigma^{\prime}=\varepsilon(g) \cdot \sigma^{\prime}$ for all $g \in G$, and $\sigma_{m}^{\prime}=\sigma_{m}$. Then there exists a G-equivariant local diffeomorphism $\Psi$ around $m$, such that $\Psi(m)=m, D \Psi(m)=I$, and $\Psi^{*} \sigma^{\prime}=\sigma$.

Proof. Write $\sigma_{t}=\sigma+t \cdot\left(\sigma^{\prime}-\sigma\right)$, so that $\sigma_{0}=\sigma, \sigma_{1}=\sigma^{\prime}, \tilde{g}^{*} \sigma_{t}=\varepsilon(g) \cdot \sigma_{t}$ for all $g \in G, t \in[0,1]$. One attempts to find local diffeomorphisms $\Psi_{t}, G$-equivariant, depending smoothly on $t$, such that $\Psi_{0}=$ identity, and $\Psi_{t}(m)=m, D \Psi_{t}(m)=I$, $\Psi_{t}^{*} \sigma_{t}=\sigma$ for all $t \in[0,1]$. Differentiating with respect to $t$ one finds that the velocity field $v_{t}$ has to satisfy the equations

$$
\left.d\left(v_{t}\right\lrcorner \sigma_{t}\right)=\sigma-\sigma^{\prime}, \quad v_{t}(m)=0, \quad D v_{t}(m)=0,
$$

for all $t \in[0,1]$. By the Poincare lemma there is a smooth 1 -form $\alpha$ such that $d \alpha=\sigma-\sigma^{\prime}, \alpha(m)=0, D \alpha(m)=0$. Let $v_{t}$ be the unique vector field such that $\left.v_{t}\right\lrcorner \sigma_{t}=\alpha$. Then $v_{t}$ depends smoothly on $t$ and satisfies (2.22). Each $\tilde{g}^{*} v_{t}, g \in G$, will again satisfy (2.22), because

$$
\begin{aligned}
\varepsilon(g) \cdot\left(\sigma-\sigma^{\prime}\right) & \left.=\tilde{g}^{*}\left(\sigma-\sigma^{\prime}\right)=\tilde{g}^{*} d\left(v_{t}\right\lrcorner \sigma_{t}\right) \\
& \left.\left.=d \tilde{g}^{*}\left(v_{t}\right\lrcorner \sigma_{t}\right)=d\left(\tilde{g}^{*} v_{t}\right\lrcorner \tilde{g}^{*} \sigma_{t}\right) \\
& \left.\left.=d\left(\tilde{g}^{*} v_{t}\right\lrcorner \varepsilon(g) \cdot \sigma_{t}\right)=\varepsilon(g) \cdot d\left(\tilde{g}^{*} v_{t}\right\lrcorner \sigma_{t}\right) .
\end{aligned}
$$

So averaging over $G$ we get a smooth $G$-invariant vector field $v_{t}$, depending smoothly on $t$, and satisfying (2.22). Integrating it we get a $G$-equivariant 1-parameter family of local diffeomorphisms $\Psi_{t}$, such that $\Psi_{t}(m)=m, D \Psi_{t}(m)=I$, and $t \mapsto \Psi_{t}^{*} \sigma_{t}$ is constant, hence equal to $\sigma$. Taking $\Psi=\Psi_{1}$, the lemma is proved.

Ignoring the $T$-action in Proposition 2.2, we have, locally, recovered the theorem of Meyer mentioned before. Moreover, the set

$$
C=\{m \in Q ; D(f \mid Q)(m)=0\}
$$

is equal to $F \cap Q$, where $F$ is the fixed point set of the $T$-action in $M$. Each connected component $C_{k}$ of $C$, which in the local coordinates of Proposition 2.2 reads as

$$
\left\{(q, p) ; p=0 \text { and } q_{j}=0 \text { whenever } \omega_{j} \neq 0\right\},
$$

is a Lagrange submanifold of some connected component $F_{j(k)}$ of $F$, the connected components $F_{j}$ of $F$ being symplectic submanifolds of $M . F_{j}$ is $\tau$-invariant if it meets 
$Q$, and then the $C_{k}$ with $j(k)=j$ are the connected components of the fixed point set for the involution $\tau$ in $F_{j}$.

We now want to prove that $f(Q)$ is equal to the convex hull of $f(C)$, which consists of only finitely many points because $f$ is obviously constant on each $C_{k}$. As in Guillemin-Sternberg [7, §5], the first step is

2.4. Lemma. For each $X \in \mathrm{t}$, the function $f_{X} \mid Q$ has a unique local maximal value.

Proof. Fix $X \in \mathrm{t}$. Let $m \in Q$ be a critical point of $f_{X} \mid Q$. From Lemma 2.1 we know that $m$ is a fixed point for the action of $\exp t X, t \in \mathbf{R}$. Replacing, in this proof, $T$ by the closure of $\{\exp t X, t \in \mathbf{R}\}$ in $T$, we get that $D(f \mid Q)(m)=0$. A glance at (2.13) and the local characterization of $Q$ shows that $m$ is a local maximum for $f_{X} \mid Q$ if and only if $m$ is a local maximum for $f_{X}$, both conditions being equivalent to $\omega_{j}(X) \leqslant 0$ for all $j$. A Morse theoretic argument gives that the set of points in $M$ where $f_{X}$ has a local maximum is connected, see Atiyah [2] and Guillemin-Sternberg [7]. So $f_{X}$ on $M$ has only one local maximal value, and therefore the same must hold for $f_{X} \mid Q$.

Now, let $\xi \in \mathrm{t}^{*}$ be a boundary point of $f(Q)$, and let $m \in Q$ be such that $f(m)=\xi$. Then

$$
\mathrm{t}_{m}=\left\{X \in \mathrm{t} ; d\left(f_{X} \mid Q\right)(m)=0\right\}
$$

is nonzero, otherwise $D(f \mid Q)(m)$ would be surjective, contradicting that $\xi$ is a boundary point. In view of Lemma 2.1 we may replace $T$ by the subtorus $\exp _{m}$, fixing $m$, and apply Proposition 2.2. Write

$$
\gamma_{m}=\sum_{j=1}^{n} \vartheta_{j} \cdot \omega_{j}, \quad \vartheta_{j} \geqslant 0,
$$

for the convex cone in $\mathrm{t}_{m}^{*}$ generated by the vectors $\omega_{j}$ of (2.13), with $t$ replaced by $t_{m}$. Then (2.13) shows that there is a neighborhood $U$ of $m$ in $Q$ and a neighborhood $V$ of $\pi_{m}(\xi)$ in $\mathrm{t}_{m}^{*}$, such that

$$
\pi_{m}(f(U))=V \cap\left(\pi_{m}(\xi)+\gamma_{m}\right) .
$$

Here $\pi_{m}$ is the projection: $\mathrm{t}^{*} \rightarrow \mathrm{t}_{m}^{*}$ obtained by restriction of linear forms to $\mathrm{t}_{m}$. This implies of course that

$$
f(U) \subset \xi+\pi_{m}^{-1}\left(\gamma_{m}\right) .
$$

Note that $\operatorname{Ker} \pi_{m}=\operatorname{Im} D(f \mid Q)(m)$. The fact that there are only finitely many possibilities for the $T_{m}$ in (2.12), and that $\mathrm{t}_{m}$ is equal to the Lie algebra of $T_{m}$, leads to $\operatorname{Im} D(f \mid Q)\left(m^{\prime}\right) \supset \operatorname{Im} D(f \mid Q)(m)$ for all $m^{\prime}$ in a neighborhood of $m$, so (2.27) can actually be strengthened to

$$
f(U)=W \cap\left(\xi+\pi_{m}^{-1}\left(\gamma_{m}\right)\right)
$$

for some neighborhood $W$ of $\xi$ in $\mathrm{t}^{*}$.

Because $\xi$ is a boundary point of $f(Q)$, we see from (2.13) and (2.29) that $\gamma_{m} \neq \mathrm{t}_{m}^{*}$, so the dual cone

$$
\delta_{m}=\left\{X \in \mathrm{t}_{m} ; \omega_{j}(X) \leqslant 0 \text { for all } j\right\}
$$


must contain nonzero $X$. However, $X \in \mathrm{t}_{m}$ means in view of (2.28) that $\langle X, f(U)\rangle \leqslant$ $\langle X, \xi\rangle$, that is, $\langle X, \xi\rangle$ is a local maximal value for $f_{X} \mid Q$. Since Lemma 2.4 implies that local maximal values are global maximal values, $\langle X, f(Q)\rangle \leqslant\langle X, \xi\rangle$ for all $X \in \delta_{m}$. That is, (2.28) can be strengthened to

$$
f(Q) \subset \xi+\pi_{m}^{-1}\left(\gamma_{m}\right) .
$$

In particular, since $\delta_{m} \neq\{0\}$, each boundary point of $f(Q)$ is on the boundary of a half-space containing $f(Q)$, so $f(Q)$ is convex. Because $f(Q)$ is compact, it is equal to the convex hull of its extremal points. But (2.29) shows that $\xi$ can only be extremal if $\mathrm{t}_{m}=\mathrm{t}$, that is $m \in C$, and $\gamma_{m}$ is a proper cone in $\mathrm{t}^{*}$. Also, (2.13) shows that $\gamma_{m}$ is constant, say equal to $\gamma\left(C_{k}\right)$, if $m$ runs along a connected component $C_{k}$ of $C$. Since a nonvoid compact convex set is equal to the intersection of the half-spaces containing it and supported at the extremal points, the local description in (2.29), (2.31) of $f(Q)$ leads to

$$
f(Q)=\bigcap_{\gamma\left(C_{k}\right) \text { a proper cone }}\left(f\left(C_{k}\right)+\gamma\left(C_{k}\right)\right) .
$$

Working on $M$ and ignoring the involution $\tau$, the above arguments also lead to

$$
f(M)=\bigcap_{\gamma\left(F_{j}\right) \text { a proper cone }}\left(f\left(F_{j}\right)+\gamma\left(F_{j}\right)\right),
$$

where the $F_{j}$ are the connected components of $F$, the fixed point set of the $T$-action on $M$, and $\gamma\left(F_{j}\right)$ is the convex cone in $\mathrm{t}^{*}$ generated by the $\omega_{j} \in \mathrm{t}^{*}$ as in (2.13), in the analogue of Proposition 2.2. disregarding the involution $\tau$. Now each $C_{k}$ was contained in an $F_{j(k)}$, of course $f\left(C_{k}\right)=f\left(F_{j(k)}\right)$. However, (2.13) shows that also $\gamma\left(C_{k}\right)=\gamma\left(F_{j(k)}\right)$. So comparing (2.32) and (2.33) we get $f(Q) \supset f(M)$. Because the other inclusion is trivial, we have proved

2.5. TheOREM. $f(Q)=f(M)$, and is equal to the convex hull of the finitely many values $f(m)$ of $f$ at points $m \in Q$ such that

(2.34) $D(f \mid Q)(m)=0$ and the convex cone $\gamma_{m}$ generated by the $\omega_{j} \in \mathrm{t}^{*}$ in (2.13) is proper.

Moreover, if $m^{\prime} \in M, D f\left(m^{\prime}\right)=0$ and the corresponding cone $\gamma_{m^{\prime}}$ is proper, then there exists $m \in Q$ such that (2.34) holds, and $f\left(m^{\prime}\right)=f(m), \gamma_{m^{\prime}}=\gamma_{m}$.

REMARK. The proof shows that the same statements are true if $Q$ is replaced by any connected component $Q_{0}$ of $Q$.

3. Tightness. We make the same assumptions as in $\S 2$, namely that $(M, \sigma)$ is a compact symplectic manifold with on it a Hamiltonian action of a torus $T$, with Hamilton functions $f_{X}, X \in \mathrm{t}$. Also, $\tau$ is a smooth involution of $M$ such that $\tau^{*} \sigma=-\sigma, \tau^{*} f_{X}=f_{X}$ for all $X \in \mathrm{t}$. In this section we prove

3.1. TheOREM. Let $C_{X}$ be the critical set of $f_{X} \mid Q, X \in \mathrm{t}$. Then

$$
\operatorname{dim} H^{*}(Q ; \mathbf{Z} / 2 \mathbf{Z})=\operatorname{dim} H^{*}\left(C_{X} ; \mathbf{Z} / 2 \mathbf{Z}\right) .
$$

We begin the proof with a reduction to the case of a circle action. By restricting to the closure of $\{\exp t X ; t \in \mathbf{R}\}$ in $T$, we may assume that $\{\exp t X ; t \in \mathbf{R}\}$ is dense in 
$T$. In view of Lemma 2.1 we have now $C_{X}=F \cap Q$, where $F$ is the fixed point set of the action of $T$ on $M$. Because there are only finitely many possibilities for the stabilizer groups $T_{m}$ in (2.12), it follows that the collection of $Y \in \mathrm{t}$ such that $C_{Y}=C_{X}$ is open and dense in $\mathrm{t}$. Because the set of $Y$, such that $t \mapsto \exp t Y$ is periodic, is dense in $\mathrm{t}$, we may assume that $t \mapsto \exp t X$ is periodic, and multiplying $X$ by a suitable factor we get $\exp X=1$. All this without changing the critical set.

Now (2.8) implies that $\tau$ maps $T$-orbits to $T$-orbits, reversing the time order on them. In particular the $T$-orbits through $m \in Q$ are $\tau$-invariant, and

$$
\tau(\exp t \tilde{X}(m))=\exp -t \tilde{X}(m)
$$

But then $\tau\left(\exp \frac{1}{2} \tilde{X}(m)\right)=\exp -\frac{1}{2} \tilde{X}(m)=\exp \frac{1}{2} \tilde{X}(m)$, that is

3.2. LemMA. $\exp \frac{1}{2} \tilde{X}$ maps $Q$ to itself, ${ }^{2}$ and thereby defines a smooth involution of $Q$.

Examples (see the remark below) show that the fixed point set in $Q$ of $\exp \frac{1}{2} \tilde{X}$, which contains $C_{X}$, is not necessarily equal to $C_{X}$ (assuming that the minimal period is equal to 1). For this reason we now define, by induction over $k \in \mathbf{N}$,

$$
Q_{(0)}=Q, \quad Q_{(k)}=\left\{m \in Q_{(k-1)} ; \exp 2^{-k} \tilde{X}(m)=m\right\} .
$$

By induction over $k$ one proves

3.3. Lemma. $\exp 2^{-k} X$ is an involution of $Q_{(k-1)}$. The $Q_{(k)}$ form a decreasing family of subspaces of $Q . Q_{(k)}$ has finitely many connected components, each of which is a smooth compact submanifold of $Q_{(k-1)}$, resp. of $Q$. There exists an $N \in \mathbf{N}$ such that $Q_{(N)}=C_{X}$.

The last statement follows because such decreasing sequences of submanifolds must stabilize, that is, there is an $N \in \mathbf{N}$ such that $Q_{(k)}=Q_{(N)}$ for all $k \geqslant N$. But $m \in Q_{(k)}$ for all $k$ implies that $\tilde{X}(m)=0$, hence $d J_{X}(m)=0$, or $m \in C_{X}$.

It is known (Floyd [4 or 5, §4]) that if $B$ is the fixed point set of a periodic transformation of prime period $p$ in a compact manifold $A$, then

$$
\operatorname{dim} H^{*}(A ; \mathbf{Z} / p \mathbf{Z}) \geqslant \operatorname{dim} H^{*}(B ; \mathbf{Z} / p \mathbf{Z}) .
$$

Lemma 3.3 therefore implies

$$
\operatorname{dim} H^{*}(Q ; \mathbf{Z} / 2 \mathbf{Z}) \geqslant \operatorname{dim} H^{*}\left(Q_{(1)} ; \mathbf{Z} / 2 \mathbf{Z}\right) \geqslant \cdots \geqslant \operatorname{dim} H^{*}\left(C_{X} ; \mathbf{Z} / 2 \mathbf{Z}\right) .
$$

But the opposite inequality $\operatorname{dim} H^{*}(Q ; R) \leqslant \operatorname{dim} H^{*}\left(C_{X} ; R\right)$, valid for general coefficient rings $R$, follows from the Bott-Morse inequalities. This proves Theorem 3.1. The idea to conclude tightness by exhibiting the critical set as the fixed point set for a periodic map has been introduced by Frankel [6] in a Kähler framework.

REMARK. If $m$ is an isolated fixed point for $\exp \frac{1}{2} \tilde{X}: Q \rightarrow Q$, then this "half-turn" is the Cartan involution around $m$ with respect to any invariant Riemannian metric. Suppose now that the isometry group of the connected component $Q_{0}$ of $Q$ acts transitively, as is the case for the real flag manifolds; see $§ 5$. Then the existence of isolated fixed points for the half-turn on $Q_{0}$ makes $Q_{0}$ into a Riemannian symmetric

\footnotetext{
${ }^{2}$ Note that $Q$ is not invariant under the $\tilde{X}$-flow. In fact $\tilde{X}(m) \in P_{m}$ where $P_{m}$ is the space complementary to $T_{m} Q$, defined in (2.10).
} 
space. For symmetric real flag manifolds the tightness of $\left.f_{X}\right|_{Q_{0}}$ was proved in Takeuchi [16, pp. 167-168] by identifying the critical set with the fixed point set of a Cartan involution. Conversely, if $Q_{0}$ is a real flag manifold which is not a symmetric space, and if $f_{X} \mid Q_{0}$ has isolated critical points (as generically is the case), then the fixed point set of $\exp \frac{1}{2} \tilde{X}$ in $Q$ cannot be equal to $C_{X}$.

4. Gradient flows. If $\beta$ is a Riemannian metric on $M$ which is $T$ - and $\tau$-invariant, then the gradient vector fields of the functions $f_{X}, X \in \mathrm{t}$, are also $T$ - and $\tau$-invariant. In particular they are tangent to the fixed point set $Q$ of $\tau$, the gradient flows leaving $Q$ invariant. The formula $\beta(u, v)=\sigma(u, J v)$ defines a tensor field $J$ on $M$ which is $T$-invariant and $\tau$-anti-invariant, and $\operatorname{grad} f_{X}$ is equal to $J$ times the Hamiltonian vector field $X$ of the function $f_{X}$.

If $J$ is an integrable almost complex structure, that is if $M$ is a complex analytic manifold with complex structure equal to $J$, then $\beta+i \sigma$ is a Kähler metric on $M$. The $T$-action consists of the holomorphic mappings, whereas the involution $\tau$ is anti-holomorphic, making $Q$ into a "real subspace" of $M$ in a strong sense. Because the automorphism group of a compact complex manifold is a complex Lie group, the action of $T$ extends to a holomorphic action of the complexification $T_{c}$ of $T$. Its Lie algebra $\mathfrak{t} \otimes \mathbf{C}$ can be written as $\mathrm{t} \oplus \mathfrak{a}$ where $\mathfrak{a}=i$ t. The exponential map is taken to be injective on a, making $A=\exp$ a into a vector subgroup of $T_{c}$, and $(t, a) \mapsto t \cdot a$ is a diffeomorphism from $T \times A$ onto $T_{c}$. Now $\operatorname{grad} f_{X}=J \cdot \tilde{X}=\widetilde{i \cdot X}$, so the gradient vector fields together make up the infinitesimal action of $A$. In particular the gradient flows commute with each other, in fact this is the only additional assumption which will be used in the sequel.

4.1. TheOrem. Let $Y$ be an A-orbit in $M$ and $F_{j}, j=1, \ldots, p$, the components of the common critical points of the functions $f_{X}, X \in \mathrm{t}$, which intersect the closure $\bar{Y}$ of $Y$ in M. Then $f(\bar{Y})$ is equal to the convex polytope $P$ with extremal points equal to $c_{j}=f\left(F_{j}\right), j=1, \ldots, p$. For each open face $\varphi$ of $P$ the inverse image $f^{-1}(\varphi)$ in $Y$ consists of a simple A-orbit, and $f$ induces a homeomorphism of $\bar{Y}$ onto $P$.

This is Theorem 2 of Atiyah [2]. That theorem was phrased in terms of $T_{c}$-orbits in $M$, but using the invariance of $f$ under the $T$-action, one readily translates it into the above statement. Because $Q$ is $A$-invariant we can apply Theorem 4.1 to the $A$-orbits in $Q$. Then Theorem 4.1 is a generalization of the corresponding statement for the real flag manifolds, due to Heckman [8, Chapter 2, Theorem 3]. The following result shows that Theorem 4.1 can be regarded as a refinement of Theorem 2.5 in the case of commuting gradient flows.

4.2. Proposition. For all $m^{\prime}$ in an open dense subset $Q^{\prime}$ of $Q$, the set of $f(m)$ such that $m \in Q, D(f \mid Q)(m)=0$, and $m$ is in the closure of the A-orbit through $m^{\prime}$, is equal to the set of extremal points of $f(Q)$.

Proof. Without loss of generality we may assume that $f(Q)=f(M)$ has a nonempty interior, that is

$$
Q^{\text {reg }}=\{m \in Q ; D(f \mid Q)(m) \text { is surjective }\}
$$

is nonvoid. 
Recall that $f\left(Q_{0}\right)=f(M)$ for any connected component $Q_{0}$ of $Q$. Now $m \notin Q_{0}^{\text {reg }}$ means that there exists $X \in \mathrm{t}, X \neq 0$, such that $m$ is a critical point for $f_{X}$. For the description of the critical set $C_{X}$ of $f_{X} \mid Q_{0}$ we may, as in the proof of Theorem 3.1, assume that $t \mapsto \exp t X$ is periodic on $M$ with minimal positive period equal to 1 when starting on $Q_{0}$. Replacing $T$ for the moment by the circle $\{\exp t X ; t \in \mathbf{R}\}$, we read off from (2.13) that

$$
C_{X}=\left\{q ; q_{j}=0 \text { if } \omega_{j}(X) \neq 0\right\}, \text { locally. }
$$

Here $\omega_{j}(X)=2 \pi \cdot k_{j}, k_{j} \in \mathbf{Z}$, and not all $k_{j}$ are even, noting that the half-turn $\exp \frac{1}{2} \tilde{X}$ of Lemma 3.2 is a nontrivial involution of $Q_{0}$. If $\operatorname{codim} C_{X}=1$, locally, then only one of the $\omega_{j}(X)$ is nonzero, hence odd. The half-turn maps $q_{j}$ to $-q_{j}$, so one side of $C_{X}$ to the other. There are only finitely many $C_{X}$ 's composing $Q_{0} \backslash Q_{0}^{\text {reg }}$. Therefore, writing

$$
T_{1 / 2}=\left\{g \in T ; \tilde{g}^{2}(m)=m \text { for all } m \in Q_{0}\right\},
$$

we get that $Q_{0}^{\text {reg }} / T_{1 / 2}$ is connected. (This argument is reminiscent of the transitivity of the action of the Weyl group on the set of Weyl chambers.)

In particular, since $f$ is $T$-invariant, $f\left(Q_{0}^{\text {reg }}\right)$ is connected. It is open; and dense in $f\left(Q_{0}\right)=f(M)$, because $Q_{0}^{\text {reg }}$ is dense in $Q_{0}, Q_{0} \backslash Q_{0}^{\text {reg }}$ being equal to the union of finitely many closed submanifolds of codimension $\geqslant 1$ of $Q_{0}$.

Since there are only finitely many possibilities for the $f(\bar{Y}), Y$ an $A$-orbit in $Q_{0}$, one has that for each $m^{\prime} \in Q_{0}$ there is a neighborhood $U$ of $m^{\prime}$ in $Q_{0}$ such that $f\left(\overline{A \cdot m^{\prime \prime}}\right) \supset f\left(\overline{A \cdot m^{\prime}}\right)$ for all $m^{\prime \prime} \in U$. Let $Q_{0}^{\prime}$ be the set of $m^{\prime} \in Q_{0}$ such that $f\left(\overline{A \cdot m^{\prime \prime}}\right)=f\left(\overline{A \cdot m^{\prime}}\right)$ for all $m^{\prime \prime} \in Q_{0}, m^{\prime \prime}$ near $m^{\prime}$. Then $Q_{0}^{\prime}$ is open and dense in $Q_{0}$. Each connected component $R$ of $Q_{0}^{\prime}$ is $A$-invariant, and $f(\bar{R})=f\left(\overline{A \cdot m^{\prime}}\right)$ for each $m^{\prime} \in R$. We shall show that $f(\bar{R})=f\left(Q_{0}\right)=f(M)$, thus completing the proof of the proposition in view of Theorem 4.1. Indeed, if $m \in Q_{0}^{\text {reg }}$ then $f$ is a diffeomorphism from $A \cdot m$ to an open subset of $\mathrm{t}^{*}$, so $f(m) \notin \partial f(\bar{R})$. Because $R$ meets the open dense subset $Q_{0}^{\text {reg }}$ of $Q_{0}$, the open set $f\left(Q_{0}^{\text {reg }}\right)$ meets $f(\bar{R})^{\text {int }}$. Since $\partial f(\bar{R}) \in f\left(Q_{0}^{\text {reg }}\right)=\varnothing$, it follows that $f(\bar{R})^{\text {int }}$ contains the connected set $f\left(Q_{0}^{\text {reg }}\right)$. But this implies that $f(\bar{R})=f\left(Q_{0}\right)$.

Choose $X \in \mathrm{t}$. The stable (or unstable) manifolds of the gradient vector field of $f_{X}$ define a decomposition of $M$ and of $Q$. These are cell decompositions if $f_{X}$ is a Morse function rather than only Bott-Morse. The stable manifolds in $Q$ are the connected components of the intersections with $Q$ of the stable manifolds in $M$. In the case of the flag manifolds, and $f_{X}$ a Morse function, the closures of the stable manifolds in $M$ are complex algebraic varieties, defining cycles (the Schubert cycles) which form a basis for the homology of $M$. The closures of the stable manifolds in $Q$ are real algebraic varieties, defining cycles modulo 2 which form a basis for the homology mod 2 of $Q$. Also, two critical points in $Q$ are connected by a gradient curve in $M$ only if they are connected by a gradient curve in $Q$. See [3, §4]. Finally it is known that the image under $f$ of both the real and the complex Schubert cycles are convex polytopes, see Heckman [8, Chapter 2, Corollary 2], and Atiyah [2, \$4].

It might be interesting to investigate which of these properties generalize to the present setting. 
5. Flag manifolds. For any connected Lie group $U$ with Lie algebra $u$, Kirillov [9] ${ }^{3}$ introduced a symplectic form on each orbit of the coadjoint action of $U$ in $\mathfrak{u}^{*}$, as follows. For $\xi \in \mathfrak{u}^{*}$, the coadjoint orbit $\theta$ of $\xi$ can be identified with $U / U_{\xi}$, where

$$
U_{\xi}=\left\{g \in U ;(\operatorname{Ad} g)^{*}(\xi)=\xi\right\},
$$

is the stabilizer of $\xi$ in $U$, which has Lie algebra

$$
\mathfrak{u}_{\xi}=\{X \in \mathfrak{u} ;(\operatorname{ad} X) *(\xi)=0\} .
$$

The symplectic form on $T_{\xi} \vartheta \cong \mathfrak{u} / \mathfrak{u}_{\xi}$ is defined by

$$
\sigma_{\xi}(X, Y)=\xi([X, Y]), \quad X, Y \in \mathfrak{u} / \mathfrak{u}_{\xi},
$$

and it is then shown that $\sigma_{\xi}$ extends to a unique $U$-invariant simplectic form $\sigma$ on $\theta$. The action of $U$ on $\theta$ is Hamiltonian (though nonabelian, see Abraham and Marsden [1] for the definitions in this general case), and the momentum mapping is equal to the inclusion mapping: $\theta \rightarrow \mathfrak{u}^{*}$. If $T$ is a torus in $U$, then its action on $\theta$ is Hamiltonian with momentum mapping equal to the projection: $\mathfrak{u}^{*} \rightarrow t^{*}$, restricted to $\theta$.

Up to coverings, these are the only symplectic manifolds with transitive Hamiltonian group actions. If $U$ is compact, then the theorem of Atiyah [2] and Guillemin and Sternberg [7] gives that the projection of the coadjoint orbit of $\xi$ in $\mathfrak{u}^{*}$ to $\mathfrak{s}^{*}$, $\mathfrak{S}=$ the Lie algebra of a maximal torus in $U$, is equal to the convex hull of the Weyl group orbit of $\xi$. As we shall see below, these coadjoint orbits are the complex flag manifolds and the convexity theorem is Kostant's for the complex case. Note that because the center of $U$ is contained in $U_{\xi}$, one may assume here that $U$ has trivial center.

Now we turn to a description of the real flag manifolds, see also [3, §2]. Let $G$ be a real connected semisimple Lie group with trivial center, and let $G=K A N$ be its Iwasawa decomposition. We may think of $G=\operatorname{Ad} G$ as a matrix group, then $K, A$, $N$ are the groups of respectively the orthogonal, diagonal with positive eigenvalues, upper triangular unipotent elements of $G$. Let $g, \mathfrak{f}, \mathfrak{a}$ be the Lie algebras of $G, K, A$ respectively. For any $H \in \mathfrak{a}$, the Ad $K$-orbit of $H$ in $g$ (actually contained in a sphere in the orthogonal complement of $\mathfrak{f}$ ) can be identified with $K / K_{H}$, where

$$
K_{H}=\{k \in K ; \operatorname{Ad} k(H)=H\}
$$

is the centralizer of $H$ in $K$. The functions

$$
f_{H^{\prime}, H}(k)=\left\langle H^{\prime}, \text { Ad } k(H)\right\rangle, \quad k \in K / K_{H}, \quad H, H^{\prime} \in \mathfrak{a}
$$

(the bilinear form here is the Killing form), can be considered as testing the orthogonal projection of $\mathrm{Ad} K(H)$ to a by linear forms on $a$.

This orthogonal projection is actually the infintesimal version of the Iwasawa projection $\pi: G \rightarrow$ a defined by

$$
x \in K \cdot \exp \pi(x) \cdot N, \quad x \in G .
$$

\footnotetext{
${ }^{3}$ The fact that the form is closed and the relation with general homogeneous symplectic manifolds were observed later by Kostant and Souriau.
} 
This projection will be applied to the $K$-orbit

$$
\left\{k \cdot \exp H \cdot k^{-1} ; k \in K\right\} \cong K / K_{H}
$$

of $\exp H$ in $G$. The full convexity theorem of Kostant now states that both the Iwasawa projection of (5.7), and its infinitesimal version applied to $\operatorname{Ad} K(H)$, have their image equal to the convex hull of the Weyl group orbit of $H$ in a. We shall only discuss the functions $f_{H^{\prime}, H}$ in (5.5), noting that Heckman [8] showed that the convexity theorem for the Iwasawa projection can be proved from its infinitesimal version by a homotopy argument.

As we shall show below, $K / K_{H}$ is a connected component of $Q$ and $f_{H^{\prime}, H}=f_{X} \mid Q$, where $Q, f_{X}$ are as in $\S \S 2,3$. The symplectic manifold $M$ is equal to a complex flag manifold $U / U_{\xi}$ as above, for a suitable $U$, resp. $\xi$, and $X$ is related to $H^{\prime}$ by a linear isomorphism. This then puts the infinitesimal version of Kostant's convexity theorem in the framework of Theorem 2.5. Moreover, in Takeuchi and Kobayashi [13] and $[3, \S 4]$, it is proved that for generic $H^{\prime}, f_{H^{\prime}, H}$ is a tight Morse function on $K / K_{H}$. So Theorem 3.1 provides a new proof for this, and extends the tightness to arbitrary $H^{\prime} \in \mathfrak{a}$.

Let $G_{\mathbf{C}}$ be the complexification of $G$, with Iwasawa decomposition

$$
G_{\mathbf{C}}=U B V
$$

(we are clearly running out of letters). Here $U$ is the maximal compact subgroup of $G_{\mathbf{C}}$, which in fact is another real form of $G_{\mathbf{C}}$. If $\tau$ is the complex conjugation of $G_{\mathbf{C}}$ around $G$, then $G$ is the connected component of 1 of the fixed point set of $\tau$ in $G_{\mathrm{C}}$. Moreover, we can arrange that $U$ is $\tau$-invariant and $K$ is the connected component of 1 of the fixed point set of $\tau$ in $U$. Similarly $B$, resp. $V$, are $\tau$-invariant and $A$, resp. $N$, are the fixed point sets of $\tau$ in $B$, resp. $V$. Of these groups only $V$ is complex, in general. In fact, the complexification $C$ of $B$ is a Cartan subgroup of $G_{\mathrm{C}}, S=C \cap U$ is a maximal torus in $\mathfrak{u}$, its Lie algebra $\mathfrak{s}$ is equal to $i . \mathfrak{b}$ if $\mathfrak{b}$ denotes the Lie algebra of $B$.

For $H \in \mathfrak{b}, U / U_{H} \stackrel{\sim}{\rightarrow} G_{\mathrm{C}} / U_{H} B V$, where $U_{H} B V$ turns out to be a complex closed subgroup of $G_{\mathrm{C}}$. It contains $C V$, which is a maximal solvable subgroup of $G_{\mathrm{C}}$, called a Borel subgroup. The Borel subgroups of $G_{\mathbf{C}}$ are all conjugate to each other. The subgroups $P_{\mathbf{C}}$ of $G_{\mathbf{C}}$ containing a Borel subgroup are called the parabolic subgroups. They are also characterized as those for which $G_{\mathrm{C}} / P_{\mathrm{C}}$ is a complex projective variety. The $G_{\mathrm{C}} / P_{\mathrm{C}}$ are the complex flag manifolds. Since up to conjugacy each parabolic subgroup of $G_{\mathrm{C}}$ is of the form $U_{H} B V$ for some $H \in \mathfrak{b}$, this exhibits the $U / U_{H}$ as the general complex flag manifolds.

Now $U_{H}=U_{\xi}$ as in (5.1), if we define $\xi \in \mathfrak{g}^{*}$ by

$$
\xi(Z)=\langle i H, Z\rangle, \quad Z \in \mathfrak{g} .
$$

This identifies the coadjoint orbits of compact connected Lie groups with the complex flag manifolds.

If $H \in \mathfrak{a} \subset \mathfrak{b}$, then $U_{H} B V$ is equal to the complexification of $P=K_{H} A N$, which therefore is called a real parabolic subgroup of $G$. As a corollary, $K / K_{H} \stackrel{\sim}{\rightarrow} G / P$ has 
$U / U_{H} \stackrel{\sim}{\rightarrow} G_{\mathbf{C}} / P_{\mathbf{C}}$ as its complexfication. Conversely, $K / K_{H}$ is equal to the connected component of $1 \cdot K_{H}$ of the fixed point set of $\tau$ in $U / U_{H}$. The $K / K_{H}$ are called the real flag manifolds. For the classical groups they can be identified with spaces of flags of linear subspaces of a vector space, isotropic with respect to the bilinear form (not necessarily symmetric or nondegenerate) of which $G$ is taken as the isometry group. In particular all (isotropic) Grassmann manifolds are included in the list of examples.

Since $\tau$ leaves the elements of a fixed, and maps $\xi=i H$ to $\overline{i H}=-i H=-\xi$, we see from (5.3) that $\tau$ is antisymplectic. On the other hand, taking for $T \subset S$ the torus in $U$ generated by $\mathrm{t}=i \mathfrak{a} \subset i \mathfrak{b}=\mathfrak{g}$, we get that $T$ acts in a Hamiltonian way on $U / U_{H}$, with Hamilton function $f_{X}$ of $\tilde{X}, X \in \mathrm{t}$, equal to $f_{H^{\prime}, H}$, taking $H^{\prime}=-i X$. In particular $f_{X}$ is $\tau$-invariant.

\section{REFERENCES}

1. R. Abraham and J. Marsden, Foundations of mechanics, 2nd ed., Benjamin, New York, 1978.

2. M. F. Atiyah, Convexity and commuting Hamiltonians, preprint, Oxford Univ. Press, London, 1981.

3. J. J. Duistermaat, J. A. C. Kolk and V. S. Varadarajan, Functions, flows and oscillatory integrals on flag manifolds and conjugacy classes in real semisimple Lie groups, preprint, Utrecht, 1981.

4. E. E. Floyd, On periodic maps and the Euler characteristics of associated spaces, Trans. Amer. Math. Soc. 72 (1952), 138-147.

5. Periodic maps via Smith theory, Seminar on Transformation Groups (A. Borel, ed.), Ann. of Math. Studies, no. 46, Princeton Univ. Press, Princeton, N. J., 1960, pp. 35-47.

6. T. Frankel, Fixed points on Kähler manifolds, Ann. of Math. (2) 70 (1959), 1-8.

7. V. Guillemin and S. Sternberg, Convexity properties of the moment mapping, preprint, M.I.T., 1981.

8. G. J. Heckman, Projections of orbits and asymptotic behaviour of multiplicities for compact Lie groups, Thesis, Leiden, 1980.

9. A. A. Kirillov, Unitary representations of nilpotent Lie groups, Uspehi Mat. Nauk 17 (1962), 57-110 = Russian Math. Surveys 17 (1962), 53-104.

10. B. Kostant, On convexity, the Weyl group and the Iwasawa decomposition, Ann. Sci. École Norm. Sup. (4) 6 (1973), 413-455.

11. K. R. Meyer, Hamiltonian systems with discrete symmetry, J. Differential Equations 41 (1981), 228-238.

12. G. D. Mostow, On a conjecture of Montgomery, Ann. of Math. (2) 65 (1957), 513-516.

13. M. Takeuchi and S. Kobayashi, Minimal embeddings of R-spaces, J. Differential Geom. 2 (1968), 203-215.

14. A. Weinstein, Symplectic manifolds and their Lagrangian submanifolds, Adv. in Math. 6 (1971), 329-346.

15. C.-T. Yang, On a problem of Montgomery, Proc. Amer. Math. Soc. 8 (1957), 255-275.

16. M. Takeuchi, Cell decomposition and Morse inequalities on certain symmetric spaces, J. Fac. Sci. Univ. Tokyo 12 (1965), 81-192.

MAThematisch InstituUt, Rijksuniversiteit Utrecht, BudapestlaAN 6, De Uithof, 3508 TA UTRECHT, THE NETHERLANDS 